\title{
Spatial fluctuations in an optical parametric oscillator below threshold with an intracavity photonic crystal
}

\author{
M. A. Garcia-March, ${ }^{1,2,3}$ M. M. De Castro, ${ }^{3}$ and R. Zambrini ${ }^{3}$ \\ ${ }^{1}$ Department of Physics, Colorado School of Mines, Golden, Colorado 80401, USA \\ ${ }^{2}$ Department of Physics, University College Cork, Cork, Ireland \\ ${ }^{3}$ Institute for Cross-Disciplinary Physics and Complex Systems, IFISC (CSIC-UIB), Palma de Mallorca 07122, Spain
}

(Received 20 December 2011; published 4 May 2012)

\begin{abstract}
We show how to control spatial quantum correlations in a multimode, degenerate, type-I optical parametric oscillator below threshold by introducing a spatially inhomogeneous medium, such as a photonic crystal, in the plane perpendicular to light propagation. We obtain the analytical expressions for all of the correlations in terms of the relevant parameters of the problem and study the number of photons, entanglement, squeezing, and twin beams. Considering different regimes and configurations, we show that it is possible to tune the instability thresholds as well as the quantumness of correlations by breaking the translational invariance of the system through a photonic-crystal modulation.
\end{abstract}

DOI: 10.1103/PhysRevA.85.053804

PACS number(s): 42.65.Yj, 42.50.Dv, 42.70.Qs

\section{INTRODUCTION}

Quantum correlations between components of a system separated spatially or temporally are part of the foundation of whole new technological fields such as quantum information and computation, quantum cryptography, and quantumenhanced metrology [1-4]. Many successful implementations have been developed in optical systems in continuous variables [2,5]. An important example is the intensity of light far from the single-photon regime. The optical parametric oscillator (OPO), a device in which a classical input (laser) beam is down converted in a nonlinear medium, operates in the many-photon regime. In these devices, the nonlinearity of the quadratic crystal allows for light mode interactions, and, therefore, it is at the origin of the generation of squeezing [6,7] and entanglement [8-10] in field quadratures [2,5] (whose spectrum is continuous). Different light modes can be distinguishable, for instance, by their polarization or frequency. Recently, special attention has been devoted also to spatial degrees of freedom where quantum correlations are displayed between cavity modes or parts of light beams [11]. Indeed, many applications have been already realized with multimode light, such as optical switching [12], quantum imaging [13-16], metrology [17], and quantum information [18,19].

A very appealing possibility is to use spatial inhomogeneities in broad area devices to control relevant quantum properties of the light. In related fields, such as linear or nonlinear classical optics, it is well known that the periodic modulation of the refractive index leads to gaps in the allowed frequencies of the electromagnetic field, which is a phenomenon known as photonic band gap [20-22]. As a result, these engineered media, namely, photonic crystals (PCs), allow one to confine and guide light, leading to many applications $[20,23,24]$. If a transverse modulation of the refractive index is considered in nonlinear cavities, this was predicted to inhibit modulation instabilities at similar wavelengths [25,26]. Recently, this prediction was confirmed experimentally [27,28] and a related phenomenon was proposed in semiconductor microcavities [29]. Modulation in dissipative systems leads also to the formation of nonlinear structures, such as different kinds of discrete cavity solitons [30]. Furthermore, the use of PCs is also at the basis of many proposals in quantum optics. Seminal papers [21] pointed to the possibility of inhibiting spontaneous emission in the PC band gaps and this was recently observed in different experiments [31-33]. As a matter of fact, the use of PCs for environment (dissipation) engineering, stemming from the presence of photonic band gaps, is the basis of intense research activity on cavity QED. In particular, non-Markovian effects have been predicted in quantum optics with structured reservoirs [34], exploring also the effects on decoherence dynamics and entanglement decay $[35,36]$.

In this work, we show the effect of a transversal modulation on quantum fluctuations and correlations in a nonlinear device where the presence of gaps is expected to inhibit quantum fluctuations. We consider a photonic-crystal optical parametric oscillator (PCOPO), that is, a multimode, degenerate OPO with a PC in the cavity, as described in Ref. [26] where the PC effect on the modulation instability was studied and compared with the homogeneous case [37]. In Ref. [38], we presented a study based on the numerical analysis of quantum fluctuations using a Langevin treatment valid both above and below threshold [39]. We showed that the quantum correlations can be tuned by means of this PC, obtaining noise reduction in field quadratures, robustness of squeezing in a wider angular range, and, most remarkably, an improvement of entanglement above threshold [38]. In this paper, we present analytical results valid below the parametric threshold and based on linear and few-mode approximations in good agreement with numerical simulations of the full model. We calculate intensity fluctuations and correlations as well as quadrature squeezing and entanglement showing the effect of the modulation. The homogeneous, multimode OPO was shown to present squeezing, entanglement, and twin-beam correlations between spatial modes below threshold [40-42] and above threshold in the presence of stable patterns or even frozen chaos [39,43]. Similar effects have been predicted in Kerr media [44] and in second-harmonic generation [45], and in recent years there have been several successful experimental realizations [13-16,46-49]. The effects of a spatial modulation discussed here for an OPO can also be generalized to these 
other nonlinear devices modified by the inclusion of an intracavity PC.

The paper is organized as follows. In Sec. II, we present the model of a PCOPO using linear and few-mode approximations for the light fluctuations below threshold giving the output signal field in terms of the input one. We also introduce a set of nonlinear Langevin equations $[38,39]$ that we numerically simulate to test our approximations. In Sec. III, we obtain an analytical expression for the intensities of the signal field, showing how the instability threshold for signal emission can be either raised or lowered by means of the PC spatial modulation. Then, in Sec. IV, we obtain the expression for different quantum correlations, such as squeezing, entanglement, and twin-beam correlations. The last section is devoted to our conclusions.

\section{FEW-MODE APPROXIMATION FOR THE PCOPO}

We consider a planar cavity filled with a nonlinear $\chi^{(2)}$ medium with one of the mirrors only partially reflecting. The pump field at frequency $2 \omega$ is down converted in a signal at frequency $\omega$, with polarization orthogonal to the pump one. This constitutes an implementation of a type-I, degenerate OPO. The input beam is a plane wave propagating in the $z$ direction (the cavity axis) with amplitude $E$, which is assumed to be real. Here, we consider the effect of the transversal inhomogeneity of the medium filling the cavity. This corresponds, for example, to the introduction of a planar photonic crystal (PC) with refractive index modulation in the plane perpendicular to the light propagation direction. A sketch of the device is provided in Fig. 1 of Ref. [38]. The intracavity dynamics of this PCOPO can be described in terms of continuous boson spatial modes $\hat{A}_{0,1}(\mathbf{x}, t)$ at frequencies $\omega_{0,1}, \mathbf{x} \in \mathbb{R}^{2}$. These operators obey equal time commutation relations [11]:

$$
\left[\hat{A}_{i}(\mathbf{x}, t), \hat{A}_{j}^{\dagger}\left(\mathbf{x}^{\prime}, t\right)\right]=\delta_{i j} \delta\left(\mathbf{x}^{\prime}-\mathbf{x}\right) .
$$

The Hamiltonian operator reads $[39,41]$

$$
\begin{aligned}
\hat{H}= & \hbar \gamma \int d^{2} \mathbf{x} \sum_{i=0,1}\left\{\hat{A}_{i}^{\dagger}\left[\Delta_{i}(\mathbf{x})-c_{i} \nabla^{2}\right] \hat{A}_{i}\right\} \\
& +i E\left(\hat{A}_{0}^{\dagger}-\hat{A}_{0}\right)+i \frac{g}{2}\left(\hat{A}_{0} \hat{A}_{1}^{\dagger 2}-\text { H.c. }\right),
\end{aligned}
$$

where the first term describes diffraction of the fields in the cavity, with $\nabla^{2}$ the Laplacian in the transverse plane and diffraction strengths $c_{1}=2 c_{0}$. The second term accounts for the interaction with the external pump $E$, while the nonlinear interaction between both modes is given by the third term, which is the coupling constant $g$ proportional to the second-order susceptibility $\chi^{(2)}$. The coefficient $\gamma$ is the cavity damping rate (introduced for convenience as a scaling).

The main difference with respect to a generic OPO $[39,41]$ is that in a PCOPO, the intracavity photonic crystal gives rise to a spatial modulation of the cavity detunings $\Delta_{0,1}(\mathbf{x})$ [38]. This constitutes a breaking of the translational symmetry of the system, with deep consequences both in the macroscopic field dynamics and in the correlations between fluctuations. In Ref. [38], numerical results about quantum effects both below and above threshold were reported, based on a simulation of the quantum-field dynamics in the $Q$ representation. From the methodological point of view, this description, as discussed in
Ref. [39], allows one to take into account the full nonlinear dynamics, with the drawback of not being amenable for analytical calculations. In the following, we introduce a simplified and approximated model that we use in order to obtain analytical results below the instability threshold.

The intracavity field operators $\hat{A}_{0}, \hat{A}_{1}$ obey the Heisenberg equation

$$
\frac{\partial \hat{A}_{j}}{\partial t}=\frac{i}{\hbar}\left[\hat{H}, \hat{A}_{j}\right]-\gamma \hat{A}_{j}+\sqrt{2 \gamma} \hat{A}_{j}^{\mathrm{in}},
$$

where the dissipative contribution characterizes such an open system, with $\sqrt{2 \gamma} \hat{A}_{j}^{\text {in }}$ incoming quantum fluctuations [50]. Due to the cubic form of the Hamiltonian (2), the dynamic equations for the operator moments form an infinite hierarchy of coupled equations, which in turn is unsuitable to handle analytically.

A commonly invoked approximation based on a system size expansion is the linearization around a macroscopic steady state leading to a dynamical evolution for the quantum fluctuations governed by a quadratic Hamiltonian [51]. Let us identify in each field operator a reference average value $A_{j}$ and a small fluctuation around it, $\hat{a}_{j}=\hat{A}_{j}-A_{j}$. The reference values $A_{j}$ are the expectation values of $\hat{A}_{j}$, and their evolution is obtained by averaging the Heisenberg equations (3) and by approximating all nonlinear terms as the product of first-order moments. This procedure leads to two classical equations whose steady state clearly depends on the regime in which the PCOPO is considered. If we consider pump values $E$ such that the PCOPO is below the instability threshold, then the signal operator expectation value is vanishing, $A_{1}=0$, independently of the presence of the PC. The equation for the average value of the pump field in this regime reduces to

$$
\partial_{t} A_{0}=-\left[1+i \Delta_{0}(\mathbf{x})-i \nabla^{2}\right] A_{0}+E,
$$

where we have introduced the scaling $\mathbf{x}^{\prime}=\mathbf{x} / \sqrt{c_{1}}$ and $t^{\prime}=\gamma t$. This scaling is used in the remainder of this article together with the scaling for the field variables described in [39]. In the following, we omit the primes to simplify notation.

In the case of homogeneous detuning $\Delta_{0}(\mathbf{x})=\Delta_{0}$, the steady-state solution of this equation is immediately found. Then, for an OPO (or a PCOPO whose modulation is only in the signal detuning), the steady state is homogeneous, $A_{0}=E /\left(1+i \Delta_{0}\right)$.

For a nonhomogeneous pump detuning, the identification of the stationary state is generally not trivial. In the following, for the sake of simplicity, we consider only one transverse dimension. Then, we assume a one-dimensional PC introduced transversally to the light propagation (see Fig. 1 of Ref. [38]). We also assume that the PC can be modeled by a sinusoidal modulation such that

$$
\Delta_{0}(x)=\Delta_{0}+M_{0} \sin \left(k_{\mathrm{p}} x\right),
$$

where $k_{\mathrm{p}}$ is the PC wave number. This is a widely used assumption that allows one to obtain analytical results because the $\mathrm{PC}$ is described by a single harmonic. Closer descriptions of the PC will require more harmonics, thus making the problem intractable analytically, while the quantum correlations obtained below will not be essentially affected by the form of the modulation. 
The steady state then satisfies, in the Fourier space,

$$
\begin{gathered}
\left(1+i \Delta_{0}+i k^{2}\right) A_{0}^{s}(k)+\frac{M_{0}}{2}\left[A_{0}^{s}\left(k-k_{\mathrm{p}}\right)\right. \\
\left.-A_{0}^{s}\left(k+k_{\mathrm{p}}\right)\right]=\delta(k) E .
\end{gathered}
$$

This gives rise to coupled-mode equations for varying $k$. We neglect terms with $|k|>k_{\mathrm{p}}$, which is a key assumption justified in Sec. II B. We then obtain for the pump field three nonvanishing modes

$$
A_{0}^{s}(x)=\sum_{k=0, \pm k_{\mathrm{p}}} A_{0}^{s}(k) e^{i k x},
$$

with

$$
\begin{gathered}
A_{0}^{s}\left(k_{\mathrm{p}}\right)=-A_{0}^{s}\left(-k_{\mathrm{p}}\right)=\frac{\frac{-M_{0}}{2} A_{0}^{s}(0)}{1+i k_{\mathrm{p}}^{2}}, \\
A_{0}^{s}(k=0)=\frac{E\left(1+i k_{\mathrm{p}}^{2}\right)}{1+i k_{\mathrm{p}}^{2}+M_{0}^{2} / 2},
\end{gathered}
$$

where we assume $\Delta_{0}=0$ without loss of generality. The steady states of the pump and (vanishing) signal fields $A_{0,1}^{s}$ are then a reference state about which the fluctuation operators $\hat{a}_{0,1}$ are defined. With a standard procedure, the exact Hamiltonian is approximated to one quadratic in these fluctuations [42,51]. The pump and signal Heisenberg equations are actually decoupled and the following dynamical equation for the signal fluctuations is obtained:

$$
\partial_{t} \hat{a}_{1}=-\left(1+i \Delta_{1}-i 2 \nabla^{2}\right) \hat{a}_{1}+A_{0}^{s} \hat{a}_{1}^{\dagger}+\sqrt{2 / \gamma} \hat{a}_{1}^{\text {in }} .
$$

To simplify notation, in the following we drop the hats of the operators and we denote the fluctuation $\hat{a}_{1}$ as $a_{1}$. The modulation on the signal will be similar to the pump one,

$$
\Delta_{1}(x)=\Delta_{1}+M_{1} \sin \left(k_{\mathrm{p}} x\right) .
$$

Notice that in general, the amplitudes of both modulations are not equal, $M_{1} \neq M_{0}$. Due to the presence of the PC, the farfield fluctuation operators $a_{1}(k)^{1}$ do not evolve independently. Different ( $k$ wave vectors or) modes are dynamically coupled,

$$
\begin{aligned}
\partial_{t} a_{1}(k, t)= & -\left(1+i \Delta_{1}+i 2 k^{2}\right) a_{1}(k, t) \\
& +\frac{M_{1}}{2}\left[a_{1}\left(k+k_{\mathrm{p}}, t\right)-a_{1}\left(k-k_{\mathrm{p}}, t\right)\right] \\
& +\sum_{n=0, \pm 1} A_{0}^{s}\left(n k_{\mathrm{p}}, t\right) a_{1}^{\dagger}\left(-k+n k_{\mathrm{p}}, t\right)+\sqrt{\frac{2}{\gamma}} a_{1}^{\text {in }}(k, t),
\end{aligned}
$$

due to the spatial modulation of both the signal detuning $\left(M_{1} \neq\right.$ $0)$ and the pump one [through the spatial harmonics $\left.A_{0}^{s}\left(n k_{\mathrm{p}}\right)\right]$. Notice that neglecting higher harmonics is equivalent to considering only $n=0, \pm 1$ in Eq. (9). Still, Eq. (9) unveils the dynamical coupling of six different modes, as discussed in Appendix A, and further approximations are needed in order to handle this model analytically.

${ }^{1}$ For the Fourier transform, we use the convention $a(k, \omega)=$ $\frac{1}{2 \pi} \int d^{2} x \frac{1}{\sqrt{2 \pi}} \int d t e^{-i(k x-\omega t)} a(x, t)$ and, for the Hermitian conjugate, $a^{\dagger}(-k,-\omega)=\frac{1}{2 \pi} \int d^{2} x \frac{1}{\sqrt{2 \pi}} \int d t e^{-i(k x-\omega t)} a^{\dagger}(x, t)$.

\section{A. Numerical simulations of fully multimode and nonlinear dynamics}

This leads us to examine the full PCOPO model in order to identify the most relevant spatial modes in different regimes. The full dynamics can be calculated by numerical simulation of Langevin equations [38,39], obtained by mapping the full master equation for the PCOPO-whose system Hamiltonian is given in Eq. (2) —onto an equation of motion for the Husimi quasiprobability distribution $Q$ in phase space $[39,50]$. This representation is then a functional of the $c$-number fields $\alpha_{i}(x)$ that are used to get the expectation values of the operators $\hat{A}_{i}(x)$ [50]. In regimes where pump intensities are not too high, the Husimi distribution $Q$ dynamics is governed by a Fokker-Planck equation, mapped in the following nonlinear Langevin equations for spatially dependent pump $\alpha_{0}$ and signal $\alpha_{1}$ fields [39]:

$$
\begin{aligned}
\partial_{t} \alpha_{0}(x, t)= & -\left\{\left[1+i \Delta_{0}(x)\right]-i \nabla^{2}\right\} \alpha_{0}(x, t) \\
& +E-\frac{1}{2} \alpha_{1}^{2}(x, t)+\xi_{0}(x, t), \\
\partial_{t} \alpha_{1}(x, t)= & -\left\{\left[1+i \Delta_{1}(x)\right]-2 i \nabla^{2}\right\} \alpha_{1}(x, t) \\
& +\alpha_{0}(x, t) \alpha_{1}^{*}(x, t)+\xi_{1}(x, t),
\end{aligned}
$$

with $\xi_{0}$ additive and $\xi_{1}$ multiplicative, phase sensitive, white noises. Notice that again the effect of the PC is enclosed in the spatial dependence of the detunings $\Delta_{0}(x)$ and $\Delta_{1}(x)$.

To study the dynamics of the system, we have simulated these equations numerically (technical details about numerical methods are given in Ref. [45]). For the OPO, without PCs, it is known that for negative signal detuning, a modulation

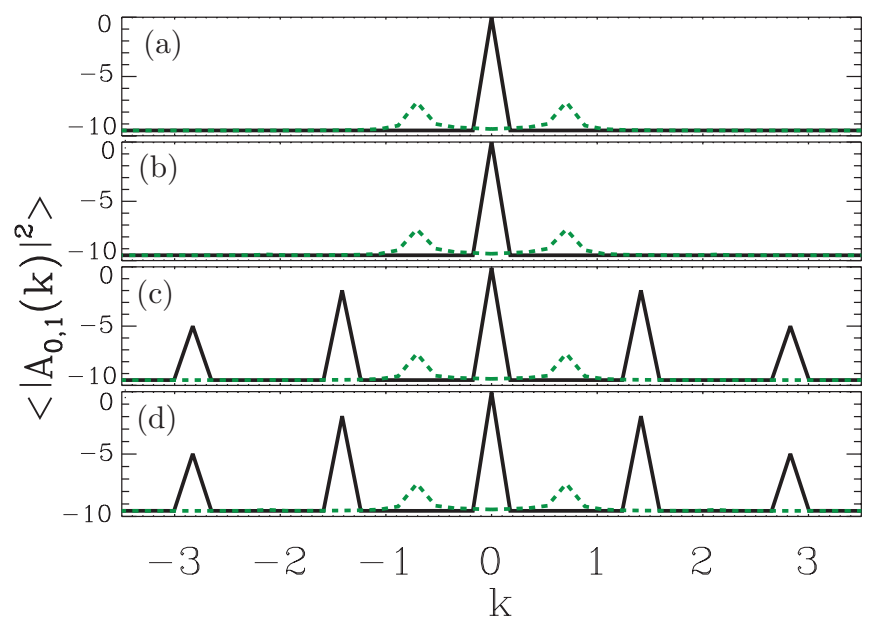

FIG. 1. (Color online) Average far fields (in logarithmic scale) obtained by numerical simulation of the full nonlinear Langevin equations. Pump is shown by black solid line and signal is shown by green dashed line. (a) corresponds to the case without $\mathrm{PC}$ and $E=0.999$, similar to the case with the PC affecting only the signal, represented in (b) for $M_{1}=0.5, M_{0}=0$, and $E=1.028$. (c) shows the case with $M_{0}=0.5, M_{1}=0$, and $E=0.931$, similar to the case where the PC affects both fields, represented in (d) for $M_{0}=M_{1}=0.5$ and $E=0.956$. The homogeneous component is always present and harmonics appear for the signal at $k_{c}=\sqrt{-\Delta_{1} / 2} \simeq 0.7$ and for the pump at $2 k_{c}$. The external pump is $0.1 \%$ smaller than its corresponding threshold. 
instability develops above the parametric threshold [37], with critical wave number $k_{c}=\sqrt{-\Delta_{1} / 2}$ (here we fix $\Delta_{1}=-1$ so that $k_{c} \simeq 0.7$ ). Below threshold, the pump is homogeneous while noisy precursors at the critical wave number are observed, with an average intensity increasing when approaching the threshold [39]. In Fig. 1(a), we show the average pump and signal far fields in the OPO, with the homogeneous and critical modes excited, respectively.

We now consider the PCOPO and in particular we focus on the case in which the PC has a band gap at the critical wave number of the OPO,

$$
k_{\mathrm{p}}=2 k_{c} \text {. }
$$

If only the signal is modulated, for $M_{0}=0$ and $M_{1}=0.5$, then the PCOPO shows average far fields similar to the case of the OPO. On the other hand, if we introduce a modulation in the pump detuning, $M_{0} \neq 0$, then the pump field develops many even harmonics, $2 k_{\mathrm{p}}, 4 k_{\mathrm{p}}, 6 k_{\mathrm{p}}, \ldots$, as shown in Figs. 1(c) and 1(d). It is also shown that the signal average far-field intensity remains unchanged in all cases.

Before we proceed to the analytical evolution of the correlation, we show here an interesting aspect of the signal fluctuations, as obtained by numerical simulations of Eqs. (10). Below threshold, noisy precursors (quantum images) dominate the dynamics, as shown in Fig. 2(a): the "preferred" spatial periodicity corresponds to the critical wave number. In the OPO, the phase of this noisy pattern is not fixed and it diffuses in space in the $x$ direction, which is dominated by the Goldstone mode, as discussed in Ref. [44]. A different behavior appears when the PC breaks the translational symmetry leading to a modulation of the pump $\left[M_{0} \neq 0\right.$, as in Fig. 2(b)]. Then the noisy pattern in the signal appears to be spatially locked and there are two $\pi$-dephased modulated modes dominating the pattern. The consequences in terms of correlations are discussed in the following sections.

\section{B. Few-mode dynamics}

From Fig. 1, we can now see that the assumption $\tilde{A}_{0}^{s}(x) \approx$ $\tilde{A}_{0}^{s}(0)+\tilde{A}_{0}^{s}\left(k_{\mathrm{p}}\right) e^{i k_{\mathrm{p}} x}+\tilde{A}_{0}^{s}\left(-k_{\mathrm{p}}\right) e^{-i k_{\mathrm{p}} x}$ [Eqs. (5)-(7)] is well justified as it takes into account the most relevant modes for the pump field: higher order harmonics at $\pm 2 k_{\mathrm{p}}$ are much smaller than the ones at $\pm k_{\mathrm{p}}$, allowing neglection of the contribution of terms with $|k|>k_{\mathrm{p}}$, i.e., modulations below the PC wavelength. On the other hand, the introduction of


FIG. 2. (Color online) Near-field evolution of the real part of signal $\alpha_{1}(x, t)$ (a) in OPO and (b) in PCOPO with PC affecting the pump, both $0.1 \%$ below the corresponding threshold. Space (abscissa) and time (ordinate) are scaled with diffraction length and cavity decay as mentioned in the text. the modulation does not have any effect on the signal, whose main components are always the modes at the critical wave number $k_{c}=k_{\mathrm{p}} / 2$. Then we restrict our analysis to the modes $k= \pm k_{\mathrm{p}} / 2$ in Eq. (9). Within this assumption, we reduce the study of the PCOPO dynamics below threshold to four coupled operator equations that in the frequency domain read

$$
L \vec{a}_{1}=\sqrt{\frac{2}{\gamma}} \vec{a}_{1}^{\text {in }},
$$

where

$$
\vec{a}_{1}=\left(a_{1}\left(k_{c}\right), a_{1}\left(-k_{c}\right), a_{1}^{\dagger}\left(-k_{c}\right), a_{1}^{\dagger}\left(k_{c}\right)\right)^{\top},
$$

and $a_{1}\left(k_{c}, \omega\right)$ and $a_{1}^{\dagger}\left(k_{c},-\omega\right)$ are denoted as $a_{1}\left(k_{c}\right)$ and $a_{1}^{\dagger}\left(k_{c}\right)$, respectively. The vector $\vec{a}_{1}^{\text {in }}$ is expressed in a similar manner. The matrix $L$ is

$$
L=\left(\begin{array}{cccc}
1-i \omega & \frac{M_{1}}{2} & -S & -\kappa S \\
-\frac{M_{1}}{2} & 1-i \omega & \kappa S & -S \\
-S^{*} & \kappa^{*} S^{*} & 1-i \omega & -\frac{M_{1}}{2} \\
-\kappa^{*} S^{*} & -S^{*} & \frac{M_{1}}{2} & 1-i \omega
\end{array}\right)
$$

From Eqs. (6) and (7), with $k_{\mathrm{p}}=2 k_{c}$, we obtain

$$
A_{0}^{s}(0)=S=\frac{E\left(1-i 2 \Delta_{1}\right)}{1-i 2 \Delta_{1}+M_{0}^{2} / 2},
$$

and $A_{0}^{s}\left( \pm k_{\mathrm{p}}\right)= \pm \kappa S$, with

$$
\kappa=\frac{-M_{0} / 2}{1-i 2 \Delta_{1}} .
$$

The output fields are obtained from the input-output formalism $\vec{A}^{\text {out }}=\sqrt{2 \gamma} \vec{A}_{1}-\vec{A}_{1}^{\text {in }}$ [52], and their dynamics (in the frequency domain $\omega$ ) is governed by

$$
\vec{a}^{\text {out }}=\left(2 L^{-1}-\mathbb{I}\right) \vec{a}_{1}^{\text {in }},
$$

where $\mathbb{I}$ is the $4 \times 4$ identity. In the following, we will concentrate on spatial quantum effects in the signal field. Therefore, we can omit without ambiguity the index 1 . To calculate different correlations in the output variables given the input ones, we need to obtain the inverse of the matrix $L$ (the expression for this inverse is given in Appendix B). This formalism is the basis of the analytical quantum correlation for the output fields discussed in the following sections.

\section{INTENSITY CORRELATIONS AND THE PARAMETRIC THRESHOLD IN THE PRESENCE OF A PC}

Let us discuss the quantum effects of introducing a PC in an OPO below threshold, starting from the intensity $\left\langle n^{\text {out }}\right\rangle=\left\langle a^{\text {out, } \dagger} a^{\text {out }}\right\rangle$ of the most intense mode $\left(k_{c}=k_{\mathrm{p}} / 2\right)$. The analytical expressions of second-order moments are given in Appendix C. In Fig. 3(a), we represent the spectral intensity, $\left\langle n^{\text {out }}\left(k_{c}, \omega\right)\right\rangle$, whose analytical expression is given in Eq. (C1), for different configurations of the PC. The effect of the PC is immediately recognized on the intensity spectrum: not only can the intensity at each frequency component be largely increased or decreased with respect to the case of the OPO, but also the maximum of the spectrum can appear shifted (away 




(b)


FIG. 3. (Color online) Intensity in frequency and time domain for fixed values of the external pump, and different configurations of the PC. In (a) we show the intensity for $E=0.92$ in the frequency domain for four different configurations. In (b) we show it in the time domain for the same pump, and for different combinations of the parameters $M_{0}, M_{1}$. In this case, the threshold is not reached for any configuration. In (c) we show that if the external pump is increased to $E=0.93$, then the intensity tends to infinity for the combinations of the parameters inside the white circle.

from $\omega=0$ ) when the pump detuning is modulated, that is, for $M_{0} \neq 0$.

The (stationary) intensity is obtained after some standard but cumbersome calculations (Appendix C) and reads

$$
\begin{aligned}
& \left\langle a^{\text {out }, \dagger}\left(k_{c}, t\right) a^{\text {out }}\left(k_{c}, t\right)\right\rangle \\
& \quad=-4|S|^{2}\left[4|S|^{2}\left|1+\kappa^{2}\right|^{2}-\left(1+|\kappa|^{2}\right)\left(4+M_{1}^{2}\right)\right] / \sigma,
\end{aligned}
$$

where the denominator $\sigma$ is

$$
\begin{aligned}
\sigma= & 16|S|^{4}\left|1+\kappa^{2}\right|^{2}-8|S|^{2}\left(1+|\kappa|^{2}\right)\left(4+M_{1}^{2}\right) \\
& +\left(4+M_{1}^{2}\right)^{2},
\end{aligned}
$$

with $S$ and $\kappa$ given in Eqs. (15) and (16), respectively.

It is easy to see that this expression reduces to the one given in Ref. [41] in the absence of the PC, when $M_{0}=M_{1}=0$, i.e., $E^{2} /\left(1-E^{2}\right)$. In a previous work, we showed that the numerical results of the full model (10) are in a good agreement with this analytical expression (see Fig. 2 of Ref. [38]), thus justifying the assumptions described above.

In general, for the PCOPO, we see that both pump and signal modulations can modify the signal emission at the most intense spatial mode $k_{c}$. In Fig. 3(b), we represent the intensity obtained from Eq. (18) as a function of $M_{0}$ and $M_{1}$ for a fixed value of the external pump, $E=0.92$. The intensity can be controlled through the PC and a strong effect is found for small modulations of the signal detuning $M_{1}$ and certain values of the modulation of the pump $M_{0}\left[M_{1} \simeq 0\right.$ and $M_{0} \simeq 0.8$ in Fig. 3(b)]. While for this value of the external pump $E$ the intensity remains finite for every combination of $M_{1}$ and $M_{0}$, this is not necessarily true when the pump is increased.

Indeed, for larger pump $E$, the average intensity in the PCOPO increases and, eventually, the threshold is reached. When approaching the parametric down-conversion threshold (coinciding with the spatial instability one), our approximation would fail and this is the basis of the divergences appearing in Eq. (18). In Fig. 3(c), we represent the intensity for an external pump $E=0.93$ and different configurations of the PC. At the edges of the white circle, the intensity tends rapidly to infinity. Then, the presence of the PC has reduced the instability associated with the threshold to a value smaller than $E=0.93$ for the points inside the white circle, while in the absence of a PC (for $M_{0}=M_{1}=0, \Delta_{0}=0$ ), the threshold is reached at $E=1$.

This is not the only possible scenario. As a matter of fact, band gaps are known to reduce spontaneous emission [20-22]. Therefore, for our choice $k_{\mathrm{p}}=2 k_{c}$, the PC is expected to cause reduction of the fluctuations that would lead to instability, inhibiting it. Indeed, the threshold can be either lowered or raised by means of the $\mathrm{PC}$, as discussed in Ref. [38]. As the signal wave number $k_{c}$ is in the band gap [25,26], inhibition of the spatial instability (lowering the threshold) is expected. On the other hand, the pump wave number is $2 k_{c}$, and therefore the $\mathrm{PC}$ introduces in the system exactly the wave number at which the instability process should occur in the pump, imprinting a nonlinear structure that favors the instability. Then a raising of the threshold can be expected. We find that these two competing mechanisms can increase or decrease the threshold depending on the relative values of the different amplitudes of the spatial detuning, $\Delta_{0}(x)$ and $\Delta_{1}(x)$.

For $M_{0}=0$, we can easily obtain an expression for the increase of the threshold with $M_{1}$. In this case, the divergence of the intensity, when Eq. (19) vanishes, leads to the threshold expression

$$
E_{\mathrm{thr}}=\sqrt{1+\left(M_{1} / 2\right)^{2}},
$$

in accordance with the results given in Ref. [26]. This curve is indeed the black curve representing the threshold in 



FIG. 4. (Color online) Minimum value of the variance for different configurations of the PC. (a) Minimum of the variance for an external pump below threshold, $E=0.92$. (b) The variance for every $\theta$ for $E$ at $5 \%$ below threshold. Here, $\phi$ is fixed to the value at which the minimum variance is obtained. (c) Minimum value of the variance for different combinations of $M_{0}$ and $E$ below threshold, when $M_{1}=0$; (d) same when $M_{1} \neq 0$ and $M_{0}=0$. This minimum is never smaller than the theoretical value in the absence of the PC, and it reaches this value at the corresponding threshold $E_{\mathrm{thr}}$, which in (c) and (d) is represented by the black solid curve.

Fig. 4(c). Moreover, in Fig. 4(b), the black curve represents the thresholds for $M_{0} \neq 0$ and $M_{1}=0$, thus showing that the threshold can be either raised or lowered with $M_{0}$. Finally, notice that if we further increase $E$, eventually reaching $E=1$, then the intensity remains finite in some regions, in accordance with the fact that the threshold is increased for some configurations of the PC.

\section{QUANTUM CORRELATIONS IN THE PRESENCE OF A PC}

As is clear from Fig. 2, there are profound effects on the fluctuations of the signal field of an OPO when including the PC modulation. Changes in the correlations between the most intense modes $a^{\text {out }}\left(k_{\mathrm{p}} / 2\right)$ and $a^{\text {out }}\left(-k_{\mathrm{p}} / 2\right)$ are then expected, and, in the following, we consider quantum effects such as squeezing, entanglement, and twin-beam correlations.

\section{A. Squeezing in the presence of PCs}

Optical parametric oscillators are well known as sources of spatial squeezing, as mentioned in Sec. I [41,42]. Two-mode squeezing appears in quadratures of the superposition of two opposite signal modes:

$$
\Sigma_{\theta \varphi}\left(k_{c},-k_{c}\right)=\left[a^{\text {out }}\left(k_{c}\right)+e^{i \varphi} a^{\text {out }}\left(-k_{c}\right)\right] e^{i \theta}+\text { H.c. },
$$

where $\theta$ is the quadrature angle and $\varphi$ is a relative phase or superposition angle. This is equivalent to a sum of (position) quadratures $\Sigma_{\theta \varphi}\left(k_{c},-k_{c}\right)=\hat{x}_{1}+\hat{x}_{2}$, where

$$
\begin{aligned}
& \hat{x}_{1}=a^{\text {out }}\left(k_{c}\right) e^{i \theta}+a^{\text {out }, \dagger}\left(k_{c}\right) e^{-i \theta}, \\
& \hat{x}_{2}=a^{\text {out }}\left(-k_{c}\right) e^{i(\theta+\varphi)}+a^{\text {out }, \dagger}\left(-k_{c}\right) e^{-i(\theta+\varphi)}
\end{aligned}
$$

are the quadratures corresponding to the mode at $k_{c}$ and $-k_{c}$, respectively.

The variance $\Delta^{2} \Sigma_{\theta \varphi}\left(k_{c},-k_{c}\right)$ can be obtained in terms of the operator moments describing the fields out of the cavity, as given in Appendix C. We remind the reader that the first-order moments of the signal field in all spatial modes vanish below threshold, so that $\Delta^{2} \Sigma_{\theta \varphi}$ is obtained from the second-order moments of the field operators.

From the analytical expression of the variance, we can find the squeezing for different combinations of $M_{0}, M_{1}$, and the pump $E$. The latter is always considered below threshold for every combination of $M_{0}$ and $M_{1}$. We observe that the level of squeezing reached depends not only on the pump $E$ but also on the values that define the PC. For example, for a fixed value of the pump $E$, it depends on $M_{0}$ and $M_{1}$, as represented in Fig. 4(a). On the other hand, this level also depends on $E$ and $M_{0}$ for fixed values of $M_{1}$ [see Fig. 4(b)], or $M_{1}$ for fixed values of $M_{0}$ [see Fig. 4(c)]. The level of squeezing depends on the distance to the threshold, which is enhanced when the system gets closer to the instability threshold. In an OPO, one can drive the system toward the instability by increasing $E$. A PCOPO permits one to control the value of the threshold through the PC, thus allowing one to control the squeezing by modifying $M_{0}$ and $M_{1}$. In Fig. 4(d), we represent how the variance varies with the quadrature angle $\theta$ for the value of the superposition angle $\phi$ at which the maximum squeezing is achieved. For all four cases, we consider that the external pump is $5 \%$ smaller than its corresponding value at the threshold for each configuration. Since the distance to the threshold is similar, the minimum variance reached for every configuration is also similar, and thus the same level of squeezing is obtained for different configurations.

In order to check the validity of our approximations, we now consider also numerical results obtained from the full nonlinear and multimode model. Figure 5 shows the variance for the fields for all of the angles and different configurations, comparing analytical and numerical results obtained after simulation of Eqs. (10), leading to intracavity correlations. The largest squeezing for an OPO in the absence of the PC is attained just at threshold between the critical modes, while in the presence of the PC this minimum value is changed. The agreement between numerical and analytical calculations is very good, as shown in Figs. 5(b) and 5(d).

In general, we find that even considering an OPO and a PCOPO at a fixed distance from the respective thresholds, the variance of $\Sigma_{\theta \varphi}$ is deeply modified by the presence of the PC. While the achieved level of squeezing is not affected by the spatial modulation, the angles at which the maximum squeezing is reached are modified. On the other hand, we mention that the results presented in Ref. [38] concerning the same device in the above threshold regime indicate strong effects such as the widening in the region of angles describing maximal squeezed states. 

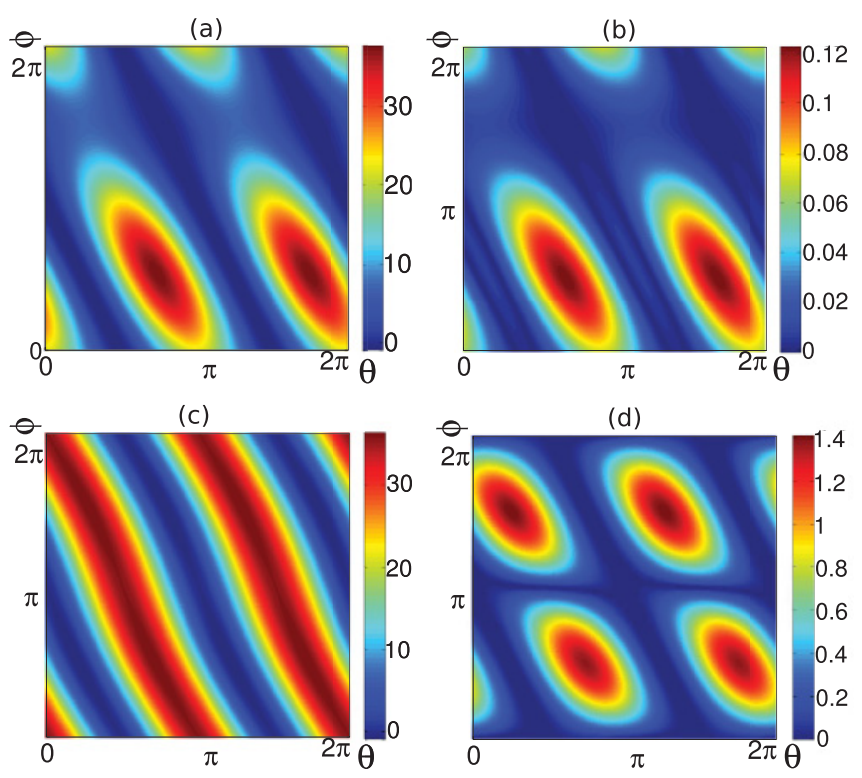

FIG. 5. (Color online) Variance for different configurations of the $\mathrm{PC}$ at all angles. The left column contains analytical results and the right one contains the absolute error with respect to the numerical calculation. All of the cases are represented for 5\% below threshold. (a) and (b) are for PCOPO with PC only affecting the pump $\left(M_{0}=\right.$ $0.5, M_{1}=0$ ) while (c) and (d) with PC only affecting the signal $\left(M_{0}=0, M_{1}=0.5\right)$.

\section{B. Entanglement measures}

In this section, we analyze the spatial entanglement between opposite modes for different parameters in order to verify if the action of the refractive index modulation disturbs entanglement measures and/or creates separable states. It is known that OPOs are useful devices giving spatially entangled states generation below threshold $[42,53,54]$. Let us analyze in this regime how the modulation introduced by the PC modifies quantum correlated states at opposite points in the far field. First, we define the (momentum) quadrature operators $\hat{p}_{i}$. These quadratures are defined similarly to $\hat{x}_{i}$, given by Eqs. (22), but with angles shifted as $\theta \rightarrow \theta-\pi / 2$ and $\varphi \rightarrow \varphi+\pi$. To establish if there is Einstein-Podolsky-Rosen (EPR) entanglement, let us define [55]

$$
\Sigma_{\theta \varphi}^{\sigma}=|\sigma| \hat{x}_{1}+\frac{1}{\sigma} \hat{x}_{2},
$$

where $\sigma$ is a real parameter. The state inseparability criterion introduced in Ref. [56] for continuous variables systems establishes that any separable quantum state characterized by a density operator $\rho$ has a lower bound on the variances sum. With the notation considered here, the inseparability criterion of Duan et al. [56] reads

$$
\Delta^{2} \Sigma_{\theta \varphi}^{\sigma}+\Delta^{2} \Sigma_{\theta+\pi / 2, \varphi+\pi}^{\sigma} \geqslant 2\left(\sigma^{2}+\frac{1}{\sigma}\right) .
$$

In Figs. 6(a)-6(d), we show this sum of variances for all of the relevant combinations of the quadrature angle $\theta$ and superposition angle $\varphi$ for four different configurations. White lines identify the regions for which the bound (24) is violated, thus corresponding to entangled states. Our analytical results show that this inseparability region is modified by a spatial modulation, even when considering OPO and PCOPO operating at the same distance from the threshold, and that the quadratures and superposition angular tolerance is slightly widened when the PC is introduced in the signal.

An alternative criterion to characterize EPR entanglement in continuous variable systems was proposed in Ref. [57]. We introduce

$$
\Delta^{2} \Sigma_{\theta \varphi}^{\lambda}=\left\langle\left(\hat{x}_{1}+\lambda \hat{x}_{2}\right)^{2}\right\rangle
$$

minimized by

$$
\lambda=\frac{-\left\langle\left(\hat{x}_{1} \hat{x}_{2}\right)\right\rangle}{\Delta^{2} \hat{x}_{2}} .
$$

According to the EPR criterion given in Ref. [57], a state is EPR entangled if

$$
\Delta^{2} \Sigma_{\theta \varphi} \Delta^{2} \Sigma_{\theta+\pi / 2, \varphi+\pi} \leqslant 1 .
$$

In Figs. 6(e)-6(h), we show the analytical calculations for this quantity for four different configurations. White lines demarcate the region for which the criterion is fulfilled and entanglement is found. Both measures show that the region where entanglement can be found is slightly widened if the $\mathrm{PC}$ is introduced in the signal. This effect is very relevant above threshold, as shown in Ref. [38].

\section{Twin-beam correlations}

Finally, we present analytical results to characterize higher order correlations not related in a trivial way to entanglement [58]. We consider twin-beam correlations [60,61] characterized by a negative value of the normal order variance, $\left\langle:(n(k)-n(-k))^{2}:\right\rangle$, where, according to the notation introduced above, $n(k)$ is the fluctuation operator $(n=\hat{N}-\langle\hat{N}\rangle)$ of the photon number $\hat{N}(k)=\left\langle\hat{A}^{\dagger}(k, t) \hat{A}(k, t)\right\rangle$. We remind the reader that the notation $\langle::\rangle$ refers to a normal ordered operator, which is obtained when no annihilation operator $\hat{a}$ ever appears to the left of any creation operator $\hat{a}^{\dagger}$. As we consider Gaussian states, all moments can be expressed as a function of second-order moments [50], giving

$$
\begin{aligned}
\langle:(n & \left.(k)-n(-k))^{2}:\right\rangle \\
= & \left\langle a^{\dagger}(k) a(k)\right\rangle^{2}+\left\langle a^{\dagger}(-k) a(-k)\right\rangle^{2}+|\langle a(k) a(k)\rangle|^{2} \\
& +|\langle a(-k) a(-k)\rangle|^{2}-2|\langle a(k) a(-k)\rangle|^{2} \\
& -2\left|\left\langle a(k) a^{\dagger}(-k)\right\rangle\right|^{2} .
\end{aligned}
$$

The nonclassical feature of twin beams follows from the negativity of the variance $\left\langle:(n(k)-n(-k))^{2}:\right\rangle<0$ and from the corresponding singularity of the $P$ representation. Indeed, the negativity of Eq. (26) corresponds to strong correlations between the twin beams; when the normally ordered variance of the intensities difference is negative, the associated fluctuations are sub-Poissonian. Fluctuations in the difference are reduced below the classical Poissonian level due to the simultaneous creation of a pair of symmetric photons $k$ and $-k$ [59-61]. Notice that terms $\langle a(k) a(k)\rangle$, in the second row of Eq. (26), vanish for an OPO in the absence of a PC or when $M_{1}=0$. This is also evident from the analytical correlations given in Appendix C. Conversely, these terms are large in PCOPO whenever the PC is affecting the signal, i.e., 
(a)

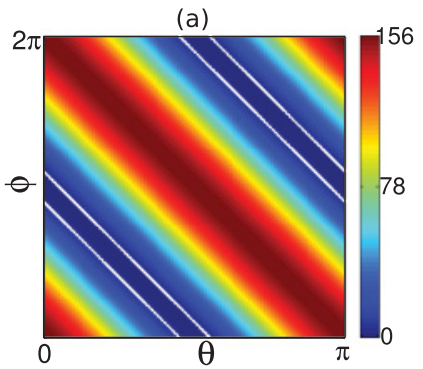

(e)



(b)



(f)

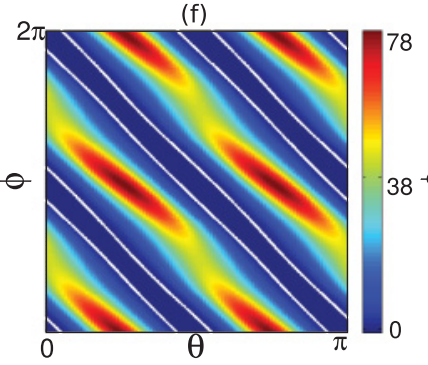

(c)

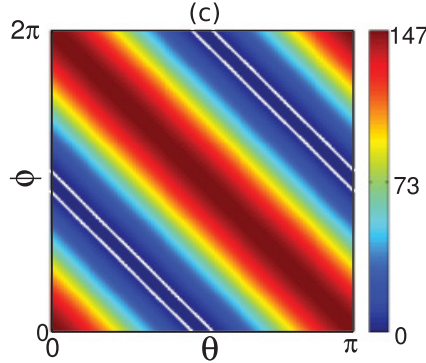

(g)

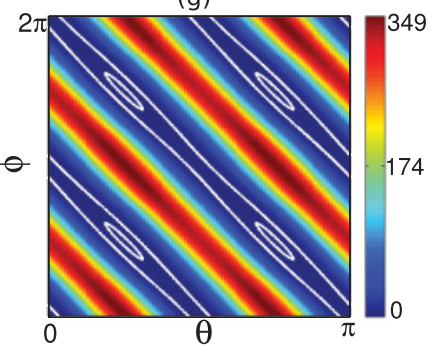

(d)

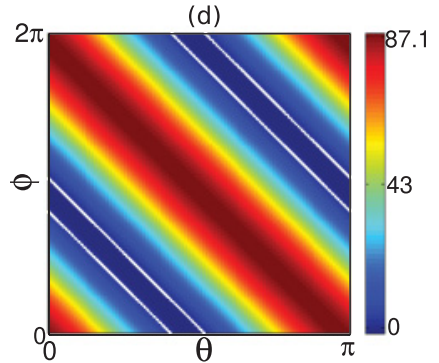

(h)

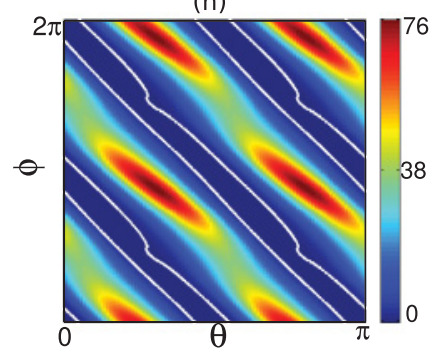

FIG. 6. (Color online) Entanglement measured with inseparability and EPR-entanglement criteria for different configurations of the PC. (a)-(d) Inseparability criteria taking $\sigma=1$ without the $\mathrm{PC}$ and for the cases $M_{0}=0.5, M_{1}=0 ; M_{0}=0, M_{1}=0.5 ; M_{0}=0.5, M_{1}=0.5$, respectively. (e)-(h) The EPR-entanglement criterion for the same cases. All of the cases are represented for 5\% below threshold. White lines demarcate the regions for which EPR entanglement is found according to both criteria.

for $M_{1} \neq 0$. Moreover, the last term $\left\langle a(k) a^{\dagger}(-k)\right\rangle$ vanishes, both for $M_{0}=M_{1}=0$ and for $M_{0}=0$ and $M_{1} \neq 0$, but it can contribute to the variance negativity for $M_{0} \neq 0$.

In particular, for $M_{0}=M_{1}=0$, Eq. (26) for the output fields yields the simple expression $[41,59]$

$$
\left\langle:\left(n\left(k_{c}\right)-n\left(-k_{c}\right)\right)^{2}:\right\rangle=-\frac{2 E^{2}}{1-E^{2}},
$$

with shot noise $\left\langle n\left(k_{c}\right)-n\left(-k_{c}\right)\right\rangle=2 E^{2} /\left(1-E^{2}\right)$. Therefore, the normalized variance is always negative and constant below threshold. This it not the case for a PCOPO, and in Fig. 7(a) we show twin-beam correlations normalized by the shot noise for a fixed value of the pump and different PC modulations. The OPO is recovered at the origin, while the twin-beam correlation is modified by the PC, becoming more classical for higher values of $M_{1}$. Let us consider now the case of modulation only in the signal detuning, that is, $M_{0}=0$ and $M_{1} \neq 0$. We find for the twin-beam correlations,

$$
\left\langle:\left(n\left(k_{c}\right)-n\left(-k_{c}\right)\right)^{2}:\right\rangle=\frac{8 E^{2}\left(4 E^{2}+M_{1}^{2}-4\right)}{\left(4-4 E^{2}+M_{1}^{2}\right)^{2}},
$$

with shot noise $\left\langle n\left(k_{c}\right)-n\left(-k_{c}\right)\right\rangle=8 E^{2} /\left(4-4 E^{2}+M_{1}^{2}\right)$. The normalized expression of the twin beams diverges for $4-4 E^{2}+M_{1}^{2}=0$, which in turn is the expression for the threshold, given by Eq. (20) [black solid curve in Fig. 7(b)]. We find that even after normalization with the shot noise, the strength of these correlations is dependent on all parameters, while in the OPO it was constant for any pump E. Moreover, in Fig. 7(b), it is clear that the correlations are degraded and can become classical below threshold (above red line). Finally, in Fig. 7(c), we present normalized twin-beam correlations when only the pump detuning is modulated. In this case, correlations remain quantum for all pump strengths below threshold, even if there is a small reduction of this quantum effect.
An interpretation of these results can be given considering the modulation effects from the microscopic point of view. A modulation in the signal (pump) detuning has the main effect of coupling different spatial modes, as is also clear from Eq. (9). In the particular case of a PC modulation with the periodicity (11), there are actually creation and destruction processes between photons at the critical tilted modes $\pm k_{c}$. Even if the nonlinearity gives rise to simultaneous creation (annihilation) of photons pairs in the signal, spatial modulation of the signal detuning leads to photons hopping between these opposite modes. In other words, there is a process of creation of one photon [say $a_{1}\left(-k_{c}\right)$ ] and destruction of one in the opposite mode $\left[a_{1}^{\dagger}\left(k_{c}\right)\right]$, as can be also seen from inspection of the Hamiltonian (2). Therefore, twin-beam correlations, due to photon pair emission, are present for small hopping rates, but when the detuning modulation of the signal is increased, the variance (28) becomes classical as the twin beams are depleted incoherently. On the other hand, for $M_{1}=0$ and modulation of the detuning of the pump field, the hopping between different pump harmonics is still detrimental, as it triggers different secondary processes besides the twin photons pair generation, but has a reduced effect.

\section{CONCLUSIONS}

We have shown the effect of an intracavity PC in a typical device displaying quantum light spatially correlated in continuous variables, as it is the degenerate, type-I OPO. Due to the translational symmetry breaking in the transverse profile, there are several effects also evident in the noisy precursors locking (Fig. 2). The PC modulation has a deep influence in the instability process, and the parametric threshold is both raised and lowered depending on the configuration (Fig. 5). As a consequence, a modification in twin-beam correlations, 
squeezing, separability, and entanglement was expected. In order to analytically evaluate these quantum correlations, we have introduced two main approximations valid below threshold, linearizing around the steady state where the signal field vanishes, and restricting our analysis to few relevant harmonics (see Fig. 2). We have considered a PC modulation (11) and five modes, $k=0, \pm k_{\mathrm{p}} / 2$ for the pump and $k=$ $\pm k_{\mathrm{p}} / 2$ for the signal. Under these approximations, we obtained good agreement between our results and the numerical solution of the full multimode nonlinear model, given by Eqs. (10).

In our prototype model, the PC leads to a sinusoidal variation on the refractive index and, therefore, of the detunings that can affect the pump, signal, or both fields, depending on the values of the PC modulations $M_{0}$ and $M_{1}$. Different configurations are described by tuning these parameters. When the PC modulates the signal field, i.e., $M_{1} \neq 0$, the instability threshold rises, which is in agreement with previous works concerning single resonant cavities and predicting pattern inhibition $[25,26]$. We demonstrate that in the presence of a parametric process, the scenario is more complex and actually the threshold can also be reduced, when $M_{0} \neq 0$, and the instability favored.

Nonclassical phenomena such as squeezing and entanglement are very sensitive to the proximity of the instability point. Therefore, the PC has deep consequences and can improve correlations at a given pump with respect to the OPO, at least when the threshold is lowered (pump modulation). Apart from this effect, all correlations have been compared in OPO and PCOPO at a fixed distance from threshold. Then we have found that squeezing (Fig. 4), separability, and EPR entanglement (Fig. 6) are preserved both in the reached values and in the width of the quadrature and superposition angle regions where these phenomena appear. Finally, we have analytically calculated twin-beam correlations (Fig. 7) when varying the PC modulations $M_{0}$ and $M_{1}$, showing that deeper effects are actually present in these intensity correlations, even at a fixed distance from the threshold, and that in general secondary processes degrade correlations. Besides, numerical simulations above threshold presented in a previous work [38] have revealed a significant enhancement of squeezing and entanglement. Overall, the PC allows one to obtain the same quantum effects at a lower energy, and one can enhance or avoid quantum properties of light just by changing the pump and/or signal spatial modulation in the cavity.

\section{ACKNOWLEDGMENTS}

We acknowledge financial support from the MICINN (Spain) and FEDER (EU) through projects FIS2007-60327 (FISICOS) and FIS2011-23526 (TIQS), from CSIC through project CoQuSys (200450E566) and from the Govern Balear through project AAEE0113/09. MAGM acknowledges support from the Spanish Ministry of Science and Education (MEC) and the US Fulbright Commission.

\section{APPENDIX A: LINEAR DYNAMICS OF PCOPO WITH ANY $K_{P}$}

If we Fourier transform in the temporal variable given by Eqs. (9) and neglect all of the terms with $|k|>k_{\mathrm{p}}$ in the signal and pump, we obtain

$$
\bar{L}_{6} \vec{a}_{1}=\sqrt{\frac{2}{\gamma}} \vec{a}_{1}^{\text {in }},
$$

where six modes are coupled between them, namely,

$$
\begin{aligned}
\vec{a}_{1}= & \left(a_{1}(k), a_{1}\left(k+k_{\mathrm{p}}\right), a_{1}\left(k-k_{\mathrm{p}}\right),\right. \\
& \left.\times a_{1}^{\dagger}(-k), a_{1}^{\dagger}\left(-k-k_{\mathrm{p}}\right), a_{1}^{\dagger}\left(-k+k_{\mathrm{p}}\right)\right)^{\top} .
\end{aligned}
$$

We use a compact notation and denote $a_{1}(k, \omega)$ simply as $a_{1}(k) ; \quad a_{1}^{\dagger}(k,-\omega)$ as $a_{1}^{\dagger}(k) ;$ and similarly for $\vec{a}_{1}^{\text {in }}$. Let us call $\eta\left(n k_{\mathrm{p}}\right)=-i \omega+\left[1+i \Delta_{1}+i 2\left(k+n k_{\mathrm{p}}\right)^{2}\right]$, $\eta^{\prime}\left(n k_{\mathrm{p}}\right)=-i \omega+\left[1-i \Delta_{1}-i 2\left(k+n k_{\mathrm{p}}\right)^{2}\right], \quad S=\tilde{S}_{0}^{s}(0)$, and $\bar{\kappa}=\left(-M_{0} / 2\right) /\left(1+i k_{\mathrm{p}}^{2}\right)$. Then we can write the matrix $\bar{L}_{6}$ (a)

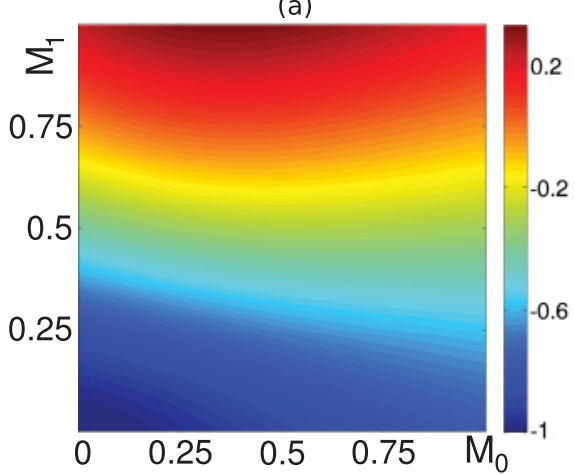

(b)

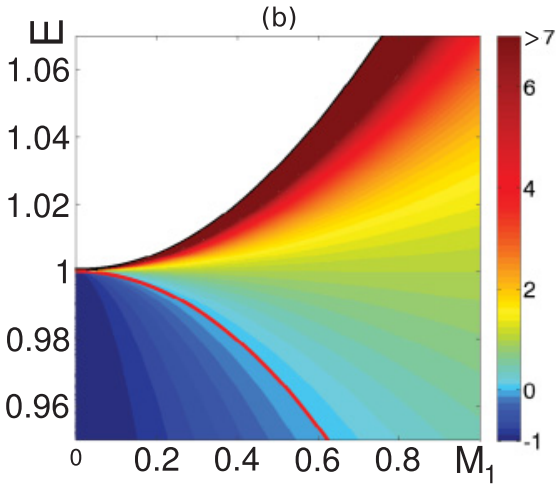

(c)

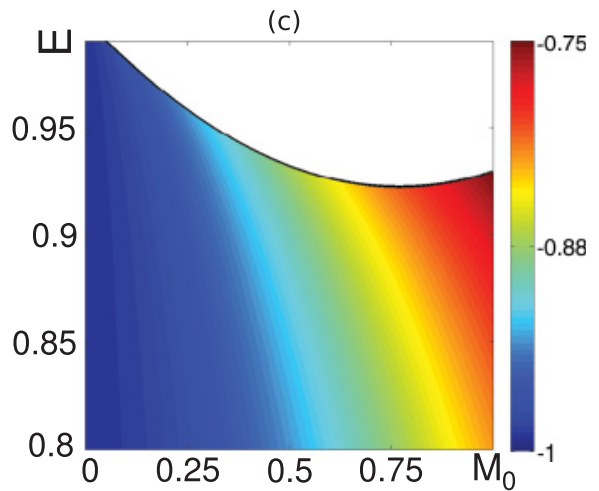

FIG. 7. (Color online) Twin-beam correlations obtained from the analytical expressions. (a) Twin-beam correlations for different configurations of the PC and $E=0.92$. (b) Twin-beam correlations below threshold for different values of $M_{1}$ and $M_{0}=0$ and normalized with the shot noise. The black solid curve represents the value of the threshold for every value of $M_{1}$. The red curve represents the change from negative to positive (classical) correlation. (c) Same as (b) for different values of $M_{0}$ and $M_{1}=0$. The black solid curve represents the value of the threshold for every value of $M_{0}$. 
as

$$
\left(\begin{array}{cccccc}
\eta(0) & -\frac{M_{1}}{2} & \frac{M_{1}}{2} & -S & \bar{\kappa} S & -\kappa S \\
\frac{M_{1}}{2} & \eta\left(k_{\mathrm{p}}\right) & 0 & -\bar{\kappa} S & -S & 0 \\
-\frac{M_{1}}{2} & 0 & \eta\left(-k_{\mathrm{p}}\right) & \bar{\kappa} S & 0 & -S \\
-S^{*} & -\bar{\kappa}^{*} S^{*} & \bar{\kappa}^{*} S^{*} & \eta^{\prime}(0) & \frac{M_{1}}{2} & -\frac{M_{1}}{2} \\
\bar{\kappa}^{*} S^{*} & -S^{*} & 0 & -\frac{M_{1}}{2} & \eta^{\prime}\left(k_{\mathrm{p}}\right) & 0 \\
-\bar{\kappa}^{*} S^{*} & 0 & -S^{*} & \frac{M_{1}}{2} & 0 & \eta^{\prime}\left(-k_{\mathrm{p}}\right)
\end{array}\right) .
$$

This matrix allows the dynamical description of the fluctuations for all modes such that $|k| \leqslant k_{\mathrm{p}}$, with continuous index $k$.

When considering the modulation $k_{\mathrm{p}}=2 k_{c}$ with $k_{c}$ as the critical wave number, few intense modes are relevant and a reduced description can be obtained, leading to the $4 \times 4$ matrix given in Eq. (14).

\section{APPENDIX B: SOLUTION OF THE INPUT-OUTPUT EQUATION}

The output field dynamics is governed by Eqs. (17). The inverse of $L$ is

$$
\frac{1}{D(\omega)}\left(\begin{array}{cccc}
U(\omega) & V(\omega) & W(\omega) & Z(\omega) \\
-V(\omega) & U(\omega) & -Z(\omega) & W(\omega) \\
W^{\prime}(\omega) & Z^{\prime}(\omega) & U^{\prime}(\omega) & V^{\prime}(\omega) \\
-Z^{\prime}(\omega) & W^{\prime}(\omega) & -V^{\prime}(\omega) & U^{\prime}(\omega)
\end{array}\right)
$$

where $D(\omega)$ is the determinant,

$$
D(\omega)=\frac{1}{2} c_{1} c_{2}+\frac{1}{4} c_{2}^{2}+|S|^{4}\left|1+\kappa^{2}\right|^{2},
$$

with $c_{1}=2|S|^{2}\left(1+\kappa^{2}\right), c_{2}=-\frac{1}{2}\left[M_{1}^{2}-4(i+\omega)^{2}\right]$. Finally, the matrix components are

$$
\begin{aligned}
U(\omega) & =-\frac{1}{2}\left(c_{1}+c_{2}\right)^{2}-(1+i \omega)\left(c_{1}+c_{2}\right)+M_{1} c_{3}, \\
U^{\prime}(\omega) & =-\frac{1}{2}\left(c_{1}+c_{2}\right)^{2}-(1+i \omega)\left(c_{1}+c_{2}\right)-M_{1} c_{3}, \\
V(\omega) & =c_{4}+\frac{M_{1}}{2}\left(c_{1}+c_{2}\right), V^{\prime}(\omega)=c_{4}-\frac{M_{1}}{2}\left(c_{1}+c_{2}\right), \\
W(\omega) & =-S\left(c_{1}+c_{2}\right), \quad W^{\prime}(\omega)=-S^{*}\left(c_{1}^{*}+c_{2}\right), \\
Z(\omega) & =-S\left(c_{1} \kappa^{*}+c_{2} \kappa\right), \quad Z^{\prime}(\omega)=S^{*}\left(c_{1}^{*} \kappa+c_{2} \kappa^{*}\right),
\end{aligned}
$$

where $c_{3}=|S|^{2}\left(\kappa-\kappa^{*}\right)$ and $c_{4}=2 c_{3}(1-i \omega)$.

\section{APPENDIX C: SECOND-ORDER MOMENTS IN FREQUENCY AND TIME DOMAINS}

We can use the expression of the output variables in terms of the input ones given in Eq. (17) to calculate different secondorder correlations in the frequency domain. For example, we can consider the correlation at different $\omega$ and the same $k$ between $a^{\text {out, } \dagger}$ and $a^{\text {out }}$. To solve the expression obtained for these correlations in terms of the input variables, we should consider that $\left\langle a^{\text {in }}(k, \omega) a^{\text {in }{ }^{\dagger}}\left(k^{\prime}, \omega^{\prime}\right)\right\rangle=\delta\left(k-k^{\prime}\right) \delta\left(\omega-\omega^{\prime}\right)$, while any other combination vanishes [this property is obtained by Fourier transforming the expression $\left\langle a^{\text {in }}(k, t) a^{\text {in, },}\left(k^{\prime}, t^{\prime}\right)\right\rangle=$ $\left.\delta\left(k-k^{\prime}\right) \delta\left(t-t^{\prime}\right)\right]$. For example, we have

$$
\begin{aligned}
& \left\langle a^{\text {out }, \dagger}\left(k_{c}, \omega\right) a^{\text {out }}\left(k_{c}, \omega^{\prime}\right)\right\rangle \\
& =4 \frac{W^{\prime}(\omega) W\left(-\omega^{\prime}\right)-Z^{\prime}(\omega) Z\left(-\omega^{\prime}\right)}{D(\omega) D\left(-\omega^{\prime}\right)} \delta(0) \delta\left(\omega-\omega^{\prime}\right) .
\end{aligned}
$$

We obtain $\delta\left(\omega-\omega^{\prime}\right)$ from the Fourier transform definition. So, $\omega^{\prime}=\omega$, due to this $\delta$ function. Notice that the matrix offers the term $a^{\text {out, } \dagger}\left(k_{c},-\omega^{\prime}\right)$, so that to obtain $a^{\text {out, } \dagger}\left(k_{c}, \omega^{\prime}\right)$, it is necessary to change the sign of the terms in the matrix. Then, for the spectral intensity, we obtain

$$
\begin{aligned}
& \left\langle a^{\text {out }, \dagger}\left(k_{c}, \omega\right) a^{\text {out }}\left(k_{c}, \omega\right)\right\rangle \\
& =4 \frac{W^{\prime}(-\omega) W(\omega)-Z^{\prime}(-\omega) Z(\omega)}{|D(\omega)|^{2}} .
\end{aligned}
$$

To obtain the intensity in the time domain, we should Fourier transform the previous expression:

$$
\begin{aligned}
& \left\langle a^{\text {out }, \dagger}\left(k_{c}, t\right) a^{\text {out }}\left(k_{c}, t\right)\right\rangle \\
& =\frac{1}{2 \pi} \int d \omega d \omega^{\prime} e^{i\left(\omega^{\prime}-\omega\right) t}\left\langle a^{\text {out }, \dagger}\left(k_{c}, \omega\right), a^{\text {out }}\left(k_{c}, \omega^{\prime}\right)\right\rangle \\
& =\frac{2}{\pi} \int d \omega \frac{W^{\prime}(-\omega) W\left(\omega^{\prime}\right)+Z^{\prime}(-\omega) Z\left(\omega^{\prime}\right)}{|D(\omega)|^{2}} .
\end{aligned}
$$

To solve this integral, we have to consider that the denominator shows eight poles of the form

$$
\omega= \pm i \pm \sqrt{-|S|^{2}+i|S|^{2}\left(\kappa-\kappa^{*}\right)-|S|^{2}|\kappa|^{2}+M_{1}^{2}},
$$

and we note that there are four in the upper part of the complex plane and four in the lower part. By performing this integral using conventional methods, we obtain (18). Any other second-order correlation can be obtained using Eq. (17), as described above. For example, all of the nonvanishing terms in the expression of the variance $\Delta^{2} \Sigma_{\theta \varphi}$ are

$$
\begin{aligned}
\left\langle\hat{x}_{1}^{2}\right\rangle= & 2 \operatorname{Re}\left\langle a\left(k_{c}\right) a\left(k_{c}\right)\right\rangle e^{2 i \theta} \\
+ & 2\left\langle a^{\dagger}\left(k_{c}\right) a\left(k_{c}\right)\right\rangle+\left[a^{\dagger}\left(k_{c}\right), a^{\dagger}\left(k_{c}\right)\right], \\
\left\langle\hat{x}_{2}^{2}\right\rangle= & 2 \operatorname{Re}\left\langle a\left(-k_{c}\right) a\left(-k_{c}\right)\right\rangle e^{2 i(\theta+\varphi)} \\
+ & 2\left\langle a^{\dagger}\left(-k_{c}\right) a\left(-k_{c}\right)\right\rangle+\left[a^{\dagger}\left(-k_{c}\right), a^{\dagger}\left(-k_{c}\right)\right], \\
\left\langle\hat{x}_{1} \hat{x}_{2}\right\rangle= & 2 \operatorname{Re}\left\langle a\left(k_{c}\right) a\left(-k_{c}\right)\right\rangle e^{i(2 \theta+\varphi)} \\
& +2 \operatorname{Re}\left\langle a^{\dagger}\left(k_{c}\right) a\left(-k_{c}\right)\right\rangle e^{i \varphi} .
\end{aligned}
$$

Finally, the Fourier transforms of all of the relevant correlations used in the definition of the variance are

$$
\begin{aligned}
\left\langle a^{\text {out }, \dagger}\left(-k_{c}, t\right) a^{\text {out }}\left(k_{c}, t\right)\right\rangle & =4 c_{3} c_{5} / \sigma, \\
\left\langle a^{\text {out }}\left(k_{c}, t\right) a^{\text {out }}\left(-k_{c}, t\right)\right\rangle & =\left[2 S\left(-2 c_{1}\right) c_{6}^{*}+c_{5} c_{6}\right] / \sigma, \\
\left\langle a^{\text {out }}\left(k_{c}, t\right) a^{\text {out }}\left(k_{c}, t\right)\right\rangle & =-\left[2 S\left(2 c_{1} c_{7}^{*}-c_{5} c_{7}\right)\right] / \sigma, \\
\left\langle a^{\text {out }, \dagger}\left(k_{c}, t\right) a^{\text {out }, \dagger}\left(-k_{c}, t\right)\right\rangle & =\left[2 S^{*}\left(-2 c_{1}^{*} c_{6}+c_{5} c_{6}^{*}\right)\right] / \sigma, \\
\left\langle a^{\text {out }, \dagger}\left(k_{c}, t\right) a^{\text {out }, \dagger}\left(k_{c}, t\right)\right\rangle & =-\left[2 S^{*}\left(2 c_{1}^{*} c_{7}-c_{5} c_{7}^{*}\right)\right] \sigma,
\end{aligned}
$$

where $\sigma$ and $c_{1}$ were defined above, $c_{5}=\left(4+M_{1}^{2}\right), c_{6}=$ $2+\kappa M_{1}$, and $c_{7}=2 \kappa-M_{1}$. 
[1] A. Galindo and M. A. Martin-Delgado, Rev. Mod. Phys. 74, 347 (2002).

[2] Quantum Information with Continuous Variables of Atoms and Light, edited by N. Cerf, G. Leuchs, and E. S. Polzik (Imperial College Press, London, 2007).

[3] N. Gisin, G. Ribordy, W. Tittel, and H. Zbinden, Rev. Mod. Phys. 74, 145 (2002).

[4] L. Maccone and V. Giovannetti, Nature Phys. 7, 376 (2011).

[5] S. L. Braunstein and P. van Loock, Rev. Mod. Phys. 77, 513 (2005).

[6] L. A. Wu, H. J. Kimble, J. L. Hall, and H. Wu, Phys. Rev. Lett. 57, 2520 (1986).

[7] H. Vahlbruch, M. Mehmet, S. Chelkowski, B. Hage, A. Franzen, N. Lastzka, S. Gossler, K. Danzmann, and R. Schnabel, Phys. Rev. Lett. 100, 033602 (2008).

[8] Z. Y. Ou, S. F. Pereira, H. J. Kimble, and K. C. Peng, Phys. Rev. Lett. 68, 3663 (1992).

[9] A. S. Villar, L. S. Cruz, K. N. Cassemiro, M. Martinelli, and P. Nussenzveig, Phys. Rev. Lett. 95, 243603 (2005).

[10] A. S. Coelho et al., Science 326, 823 (2009).

[11] M. I. Kolobov, Rev. Mod. Phys. 71, 1539 (1999).

[12] A. M. C. Dawes et al., Science 308, 672 (2005).

[13] V. Boyer et al., Science 321, 544 (2008).

[14] A. M. Marino et al., Nature (London) 457, 859 (2009).

[15] J. Janousek et al., Nature Photon. 3, 399 (2009).

[16] G. Brida et al., Nature Photon. 4, 227 (2010).

[17] N. Treps et al., Science 301, 940 (2003).

[18] M. N. O’Sullivan-Hale, I. A. Khan, R. W. Boyd, and J. C. Howell, Phys. Rev. Lett. 94, 220501 (2005).

[19] M. Lassen, V. Delaubert, J. Janousek, K. Wagner, H. A. Bachor, P. K. Lam, N. Treps, P. Buchhave, C. Fabre, and C. C. Harb, Phys. Rev. Lett. 98, 083602 (2007).

[20] J. D. Joannopoulos, S. G. Johnson, J. N. Winn, and R. D. Meade, Photonic Crystals: Molding the Flow of Light, 2nd ed. (Princeton University Press, Princeton, NJ, 2008).

[21] E. Yablonovitch, Phys. Rev. Lett. 58, 2059 (1987).

[22] S. John, Phys. Rev. Lett. 58, 2486 (1987).

[23] K. Busch, G. von Freymann, S. Linden, S. F. Mingaleev, L. Tkeshelashvili, and M. Wegener, Phys. Rep. 444, 101 (2007).

[24] P. St. J. Russell, Science 299, 358 (2003).

[25] D. Gomila, R. Zambrini and G- L. Oppo, Phys. Rev. Lett. 92, 253904 (2004).

[26] D. Gomila and G- L. Oppo, Phys. Rev. E 72, 016614 (2005).

[27] N. Marsal, D. Wolfersberger, M. Sciamanna, G. Montemezzani, and D. N. Neshev, Opt. Lett. 33, 2509 (2008); A. A. Sukhorukov, N. Marsal, A. Minovich, D. Wolfersberger, M. Sciamanna, G. Montemezzani, and D. N. Neshev, ibid. 35, 3568 (2010).

[28] B. Terhalle, N. Radwell, P. Rose, C. Denz, and T. Ackemann, Appl. Phys. Lett. 93, 151114 (2008).

[29] A. V. Gorbach and D. V. Skryabin, Phys. Rev. B 82, 125313 (2010).

[30] U. Peschel, O. Egorov, and F. Lederer, Opt. Lett. 29, 1909 (2004).

[31] S. Noda, M. Fujita, and T. Asano, Nature Photon. 1, 449 (2007), and references therein.

[32] P. Lodahl, A. F. van Driel, I. S. Nikolaev, A. Irman, K. Overgaag, D. Vanmaekelbergh, and W. L. Vos, Nature (London) 430, 654 (2004).
[33] M. Fujita, S. Takahashi, Y. Tanaka, T. Asano, and S. Noda, Science 308, 1296 (2005).

[34] P. Lambropoulos, G. M. Nikolopoulos, T. R. Nielsen, and S. Bay, Rep. Prog. Phys. 63, 455 (2000).

[35] J. Piilo, S. Maniscalco, K. Härkönen, and K. A. Suominen, Phys. Rev. Lett. 100, 180402 (2008).

[36] B. Bellomo, R. Lo Franco, and G. CompagnoPhys. Rev. Lett. 99, 160502 (2007).

[37] G. L. Oppo, M. Brambilla, and L. A. Lugiato, Phys. Rev. A 49, 2028 (1994).

[38] M. M. de Castro, M. A. Garcia-March, D. Gomila, and R. Zambrini Phys. Rev. A 84, 033832 (2011).

[39] R. Zambrini, S. M. Barnett, P. Colet, and M. San Miguel, Eur. Phys. J. D 22, 461 (2003).

[40] L. A. Lugiato and A. Gatti, Phys. Rev. Lett. 70, 3868 (1993).

[41] A. Gatti, H. Wiedemann, L. A. Lugiato, I. Marzoli, G. L. Oppo, and S. M. Barnett, Phys. Rev. A 56, 877 (1997).

[42] R. Zambrini, A. Gatti, L. Lugiato and M. San Miguel, Phys. Rev. A 68, 063809 (2003).

[43] I. Perez-Arjona et al., Europhys. Lett. 74, 247 (2006).

[44] R. Zambrini, M. Hoyuelos, A. Gatti, P. Colet, L. Lugiato and M. San Miguel, Phys. Rev. A 62, 063801 (2000).

[45] M. Bache, P. Scotto, R. Zambrini, M. San Miguel, and M. Saffman, Phys. Rev. A 66, 013809 (2002).

[46] B. Chalopin, F. Scazza, C. Fabre, and N. Treps, Phys. Rev. A 81, 061804(R) (2010).

[47] M. Lassen and G. Leuchs, and U. L. Andersen, Phys. Rev. Lett. 102, 163602 (2009).

[48] V. Boyer, A. M. Marino, and P. D. Lett, Phys. Rev. Lett. 100, 143601 (2008).

[49] B. Chalopin, F. Scazza, C. Fabre, and N. Treps, Opt. Express 19, 4405 (2011).

[50] C. W. Gardiner and P. Zoller, Quantum Noise (Springer, Berlin, 1991).

[51] H. Carmichael, An Open Systems Approach to Quantum Optics (Springer, Berlin Heidelberg, 1993).

[52] M. J. Collett and C. W. Gardiner, Phys. Rev. A 30, 1386 (1984).

[53] A. Gatti, L. A. Lugiato, K. I. Petsas, and I. Marzoli, Europhys. Lett. 46, 461 (1999); A. Gatti, K. I. Petsas, I. Marzoli, and L. A. Lugiato, Opt. Commun. 179, 591 (2000).

[54] L. Lopez, B. Chalopin, A. R. de la Souchere, C. Fabre, A. Maitre, and N. Treps, Phys. Rev. A 80, 043816 (2009).

[55] A. Einstein, B. Podolsky, and R. Rosen, Phys. Rev. 47, 777 (1935).

[56] L.-M. Duan, G. Giedke, J. I. Cirac, and P. Zoller, Phys. Rev. Lett 84, 2722 (2000).

[57] M. D. Reid and P. D. Drummond, Phys. Rev. Lett 60, 2731 (1988).

[58] G. L. Giorgi, F. Galve, and R. Zambrini, Int. J. Quantum Inf. 9, 1825 (2011).

[59] R. Zambrini and M. San Miguel, Phys. Rev. A 66, 023807 (2002).

[60] D. F. Walls and G. J. Millburn, Quantum optics, 2nd ed. (Springer-Verlag, Berlin, 1995).

[61] A. Heidmann, R. J. Horowicz, S. Reynaud, E. Giacobino, C. Fabre, and G. Camy, Phys. Rev. Lett. 59, 2555 (1987). 